\title{
La corrida, un acte de cruauté certes, mais conforme à la constitution française
}

Jean-Marc Neumann *.

Introduction

Le Conseil Constitutionnel a rendu en date du 21 septembre sa décision quant à I'inconstitutionnalité soulevée dans le cadre d'une QPC 'par deux associations des dispositions de l'alinéa 7 de l'article 521-1 du code pénal (qui réprime les actes de cruauté contre les animaux) prévoyant une exception au profit des "courses de taureaux lorsqu'une tradition locale ininterrompue peut être invoquée ".

Ainsi que de nombreux éléments permettaient, hélas, de le craindre, la décision qui a été rendue confirme la constitutionnalité de la disposition attaquée et permet la poursuite des corridas dès lors qu'une tradition locale ininterrompue peut être invoquée.

Après avoir rappelé l'origine du contentieux soulevé par I'ASSOCIATION COMITE RADICALEMENT ANTI-CORRIDA EUROPE et I'ASSOCIATION DROITS DES ANIMAUX (II), nous rappellerons synthétiquement l'ensemble de la procédure jusqu'à la saisine du Conseil Constitutionnel (III) avant d'examiner et de commenter la décision rendue par ce dernier (IV) et de conclure sur les conséquences de cette décision (V).

\footnotetext{
* directeur des affaires juridiques d'un groupe industriel international basé en France. Il a obtenu une maîtrise de droit des affaires de I'Université de Lille et d'un diplôme universitaire en terminologie juridique anglaise et la traduction de l'Institut de droit comparé de Strasbourg.

${ }^{1}$ QPC : Question prioritaire de constitutionnalité. Permise depuis la réforme constitutionnelle du 23 juillet 2008 ,il s'agit d'une procédure d'examen par voie d'exception de la constitutionnalité des lois permettant au justiciable français de lui permettre de faire valoir ses droits résultant de la Constitution.
} 


\section{L'origine du contentieux}

En janvier 2011, la Direction Générale des Patrimoines du ministère de la culture et de la communication (ministère dirigé à l'époque par Frédéric Mitterrand) avait procédé (sous la pression exercée par des milieux tauromachiques relayés par leurs appuis politiques au plus niveau) à l'inscription, en application de l'article 12 de la convention de I'UNESCO pour la sauvegarde du patrimoine culturel immatériel, de la corrida au patrimoine immatériel de la France.

Cette inscription, faite sans la moindre concertation, devait, fort légitimement, soulever de très nombreuses et vives réactions et indigner tous les opposants à la corrida. Ce n'était-ce pas fortuitement que cette inscription s'était faite en catimini ; la volonté délibérée du gouvernement de l'époque et des milieux tauromachiques était précisément d'éviter tout débat et de prendre de court les opposants à la corrida. Dans son remarquable article " La corrida aux portes du patrimoine culturel immatériel de l'humanité ?" 2, le professeur Jean-Pierre Marguénaud a cité le journal espagnol "El Mundo" qui, dans sa livraison du 03 juin 2011, avait rapporté les propos du directeur de l'Observatoire national des cultures taurines selon lequel "Ce que nous avons obtenu en France l'a été en travaillant tapis dans l'ombre". Jean-Pierre Marguénaud précise également dans son article que le directeur de la Direction Générale du Patrimoine "aurait été membre fondateur et administrateur de l'Observatoire national des cultures taurines"...

Une concertation sur un thème aussi passionnel aurait suscité un très vif débat, ce qui aurait rendu l'inscription de la corrida au patrimoine immatériel français plus difficile ou qui, à tout le moins, en aurait retardé la mise en œuvre.

\footnotetext{
${ }^{2}$ http://www.anticorrida.com/IMG/pdf/La_corrida_aux_portes_du_patrimoine_culturel_immateriel_1_1_.pdf
} 
Il convient de rappeler qu'une proposition de loi ${ }^{3}$ avait été antérieurement enregistrée au bureau du Président de l'Assemblée Nationale en date du 13 juillet 2010 demandant la suppression de l'alinéa 7 de l'article 521-1 du code pénal, ce qui montrait qu'il y avait nécessité pour les partisans de la corrida d'avancer vite et de surprendre les anti-corridas en attaquant par un angle original inattendu.

L'inscription au patrimoine immatériel français devait, dans l'esprit de ceux qui en était à l'origine, être une première étape avant l'inscription ultérieurement à la liste du patrimoine culturel immatériel de l'humanité.

Bref, inscrire la corrida au patrimoine immatériel de la France, ainsi que l'a fait le ministère de la culture, dans la plus grande opacité, sans aucun dialogue au préalable avec toutes les parties concernées, revenait, pour reprendre une image chère aux aficionados, à agiter le chiffon rouge et séparer encore davantage les points de vue des partisans et opposants à la corrida. Les évènements qui suivirent l'ont montré.

C'est dans ce contexte particulier, que deux associations ont décidé, pour reprendre une expression très imagée et appropriée au sujet, "de prendre le taureau par le cornes" et d'engager une procédure devant le tribunal administratif de Paris.

\section{La procédure jusqu'à la saisine du Conseil Constitutionnel}

L'ASSOCIATION COMITE RADICALEMENT ANTI-CORRIDA EUROPE et I'ASSOCIATION DROITS DES ANIMAUX ont saisi le tribunal administratif de Paris d'un recours tendant à I'annulation de la décision par laquelle le ministre de la culture et de la communication avait inscrit, en application de l'article 12 de la convention de I'UNESCO pour la sauvegarde du patrimoine culturel immatériel, la corrida au patrimoine immatériel de la France et de la décision implicite par laquelle le ministre a rejeté le recours gracieux dirigé contre cette décision. Le mémoire des requérantes fut enregistré le 13 octobre 2011 par le greffe.

Par ordonnance du 5 décembre 2011, la présidente de la 7ème section du tribunal administratif de Paris, avant qu'il soit statué sur la requête avait décidé de transmettre au Conseil d'État la question de la conformité aux droits et libertés garantis par la Constitution des dispositions de l'article 521-1 du code pénal.

\footnotetext{
${ }^{3}$ Proposition de loi du 13 juillet 2010 : http://www.anticorrida.com/IMG/pdf/PPL_2735.pdf
} 
Le Conseil d'État, après s'être assuré que la disposition contestée était bien applicable au litige qu'elle n'avait pas déjà été déclarée conforme à la Constitution dans les motifs et le dispositif d'une décision du Conseil Constitutionnel et que la question était nouvelle ou présentait un caractère sérieux, considérant que " le moyen tiré de ce que, en prévoyant que les faits qu'elle incrimine ne sont pas pénalement sanctionnés lorsqu'ils sont commis selon des pratiques traditionnelles locales, elle serait contraire aux droits et libertés garantis par la Constitution, et notamment au principe d'égalité devant la loi garanti par l'article 6 de la Déclaration des droits de l'homme et du citoyen du 26 août 1789, soulève une question présentant un caractère sérieux " décida le 20 juin $2012{ }^{4}$ de renvoyer la question soulevée au Conseil Constitutionnel. Ce dernier fut saisi le 21 juin 2012.

Les associations requérantes, se souvenant que l'inscription de la corrida au patrimoine immatériel de la France était intervenue sous l'ère du Président Sarkozy (devenu membre de droit à vie du Conseil Constitutionnel comme tous les anciens Présidents de la République) avaient adressé une demande de récusation de $\mathrm{M}$. Sarkozy enregistrée le 11 juillet 2012 .La demande de récusation fut acceptée et confirmée par le secrétariat du Conseil Constitutionnel.

\section{Examen et commentaires de la décision rendue par le Conseil Constitutionnel}

Le Conseil constitutionnel a été saisi afin qu'il se prononce sur la conformité aux droits et libertés que la Constitution garantit de l'article 521-1 du code pénal. Ainsi que le prévoient les textes, la décision devait intervenir dans les 3 mois de la saisine.

Très rapidement après sa saisine, le Premier Ministre, Jean-Marc Ayrault, a exprimé sa position sur la QPC selon lettre du 13 juillet $2012{ }^{5}$ adressée aux associations requérantes. Cette lettre manifestement montre un désaccord profond (ce qui n'est pas surprenant au demeurant) avec l'analyse juridique telle que présentée par les deux associations.

Dans sa lettre, les services du Premier Ministre rappelle l'historique de la dérogation instituée par l'alinéa 7 de l'article 521-1 du code pénal et formule diverses "observations" justifiant la tolérance instituée en faveur des courses de taureaux qui méritent d'être citées ici.

\footnotetext{
${ }^{4}$ Conseil d'État - décision du 20 juin 2012 N 357798

${ }^{5}$ lettre du 13 juillet 2012 adressée "Pour le Premier ministre et par délégation, La chef du service de la législation et de la qualité du droit Florence Dubois-Stévant"

http://www.les-taureaux-voteront.com/corrida/premiere-attaque-du-premier-ministre-contre-notre-qpc
} 
La lettre rappelle que" le législateur a estimé qu'il était d'intérêt général de consacrer dans la loi la tolérance dont elles bénéficiaient en pratique, en instaurant une exception à l'incrimination subordonnée à l'existence d'une tradition ininterrompue. A cette fin, le législateur a modifié la loi Grammont, par la loi du 24 avril 1951, en disposant qu'elle ne serait pas applicable aux courses de taureaux "lorsqu'une tradition ininterrompue peut être invoquée ".Ainsi qu' il ressort des travaux préparatoires, cette disposition a été adoptée par un souci d'apaisement politique et social, tenant compte de l'attachement constant de certaines populations aux courses de taureaux. L'« intérêt même de l'ordre public " a été expressément considéré (Conseil de la République, séance du 10 avril 1951, rapport de Jean Biatarana, annexe $n^{\circ}$ 241).Il a été ainsi relevé que "tous les efforts des pouvoirs publics, en vue d'essayer d'empêcher les courses de taureaux, [étaient] restés vains, au point de se heurter parfois à de véritables révoltes " (Assemblée nationale, séance du 26 juillet 1950, rapport de Joseph Defos du Rau, annexe $n^{\circ}$ 10764).Le législateur a confirmé cette exception lorsqu'il a institué, dans la loi n 63-1143 du 19 novembre 1963 relative à la protection des animaux, le délit d'acte de cruauté commis envers un animal domestique ou apprivoisé ou tenu en captivité (article 453 du code pénal devenu l'article 521-1), les mauvais traitements étant désormais constitutifs d'une contravention réprimée par les dispositions de l'article R. 38 du code pénal issues du décret $n^{\circ}$ 59-1051 du 7 septembre 1959 qui abroge la loi Grammont (article R.38 devenu l'article R. 654-1).Ce "régime de la tolérance maintenue ", selon les termes du rapporteur à l'Assemblée nationale, Max Moras (séance du 12 juillet 1961), a été considéré comme une "thèse conciliatrice " (Jean-Marie Commenay; même séance), se situant entre l'interdiction totale et l'extension généralisée à l'ensemble du territoire de l'exception d'incrimination.Le maintien de ce régime répond au même souci d'apaisement politique qu'en 1951." 
La lettre, par ailleurs, précise que "L'article 521-1 du code pénal ne méconnaît pas le principe de l'égalité devant la loi." Les dispositions contestées instituent deux différences de traitement cumulatives : d'une part, selon que les animaux participent à des courses de taureaux ou non ; d'autre part, selon que les courses de taureaux résultent de traditions locales ininterrompues ou non.ll ressort de la jurisprudence du Conseil constitutionnel que «le principe d'égalité ne s'oppose ni à ce que le législateur règle de façon différente des situations différentes ni à ce qu'il déroge à l'égalité pour des raisons d'intérêt général pourvu que, dans l'un et l'autre cas, la différence de traitement qui en résulte soit en rapport avec l'objet de la loi qui l'établit» (v., demièrement, la décision n²012-259 QPC du 29 juin 2012)."

Le Premier Ministre précise en outre que " En l'occurrence, il n'appartient pas au Conseil constitutionnel de substituer son appréciation à celle du législateur sur les motifs d'intérêt général qu'il a ainsi retenus pour justifier une exception à des dispositions réprimant les sévices et les actes de cruautés relatifs à certains animaux, dès lors qu'une telle exception ne prive par elle-même de garantie légale aucune exigence constitutionnelle" pour conclure que "Le grief tiré de la méconnaissance du principe de l'égalité devant la loi est par conséquent infondé" et que "Pour l'ensemblede ces raisons, j'ai l'honneur d'inviter le Conseil constitutionnel à déclarer conforme à la Constitution l’article 521-1 du code pénal."

Une seconde lettre sera envoyée par le Premier Ministre le 30 juillet 2012 aux associations requérantes ${ }^{6}$ et en date du 10 septembre $2012^{7}$ le Président de la République précisera "Il a également été pris note de vos remarques au sujet des récentes décisions du Conseil d'État relatives à la constitutionnalité de cette mesure.

\footnotetext{
${ }^{6}$ http://www.les-taureaux-voteront.com/corrida/deuxieme-attaque-du-premier-ministre-contre-notre-qpc
} 
A cet égard, vous le savez, le Chef de l'État ne peut, sans porter atteinte à l'indépendance de l'autorité judiciaire dont il est le garant, intervenir dans une procédure, émettre une appréciation sur les décisions rendues ou en modifier la portée."

S'est ajoutée à la prise de position pour le moins dénuée de toute ambigüité du Premier Ministre, une prise de position individuelle, à quelques heures du délibéré, d'un éminent membre du gouvernement Ayrault, en l'occurrence Manuel Valls, le ministre de l'intérieur, d'origine espagnole, qui s'est déclaré résolument favorable au maintien de la corrida en déclarant au micro de la chaine d'information BFMTV " Moi, vous savez que c'est quelque chose que j'aime".

C'est dans cet environnement extrêmement hostile aux requérantes, que les "sages" du Conseil Constitutionnel ont statué.

L'on sentait bien qu'une déclaration d'inconstitutionnalité de l'alinéa 7 de l'article 5211 du code pénal relevait quasiment de la mission impossible. Jean-Pierre Marguénaud l'avait également très bien senti et craignait les effets dévastateurs d'une décision de rejet lorsque, se référant à la QPC dans son article, il écrivit "QPC qui dans la lumière des projecteurs, pourrait, médiatiquement, entraîner un terrible effet boomerang". L'affaire fit l'objet d'une audience publique le 11 septembre 2012 (la vidéo de l'audience est accesible sur le site internet du Conseil Constitutionnel ${ }^{8}$ ) devant le Conseil Constitutionnel et fut mise en délibéré au 21 septembre 2012.

\footnotetext{
${ }^{7}$ http://www.les-taureaux-voteront.com/corrida/neutralite-du-president-de-republique

${ }^{8}$ http://www.conseil-constitutionnel.fr/conseil-constitutionnel/francais/videos/2012/septembre/affaire-n-2012-271qpc.115524.html
} 
L'attente fut longue. Chaque camp était dans l'espoir. L'espoir pour les pro-corridas que celle-ci soit maintenue. L'espoir pour les anti-corridas que l'exception instituée par l'alinéa 7 de l'article 521-1 du code pénal soit déclarée contraire à la constitution.

Le vendredi 21 septembre, tous les médias étaient sur la brèche et attendaient impatiemment la décision des sages.

La décision ${ }^{9}$ fut annoncée très tôt dans la matinée et, sans surprise, se révéla défavorable aux requérants. La nouvelle fit rapidement la une de tous les journaux et sites d'informations.

Le Conseil Constitutionnel décide (Article 1er.) que "La première phrase du septième alinéa de l'article 521-1 du code pénal est conforme à la Constitution."

Les sages rappellent que "le premier alinéa de l'article 521-1 du code pénal réprime notamment les sévices graves et les actes de cruauté envers un animal domestique ou tenu en captivité ; que la première phrase du septième alinéa de cet article exclut l'application de ces dispositions aux courses de taureaux ; que cette exonération est toutefois limitée aux cas où une tradition locale ininterrompue peut être invoquée " et qu'en procédant à une exonération restreinte de la responsabilité pénale, le législateur a entendu que les dispositions du premier alinéa de l'article 5211 du code pénal ne puissent pas conduire à remettre en cause certaines pratiques traditionnelles qui ne portent atteinte à aucun droit constitutionnellement garanti ; que l'exclusion de responsabilité pénale instituée par les dispositions contestées n'est applicable que dans les parties du territoire national où l'existence d'une telle tradition ininterrompue est établie et pour les seuls actes qui relèvent de cette tradition " et considèrent que " par suite, la différence de traitement instaurée par le législateur entre agissements de 
même nature accomplis dans des zones géographiques différentes est en rapport direct avec l'objet de la loi qui l'établit ; qu'en outre, s'il appartient aux juridictions compétentes d'apprécier les situations de fait répondant à la tradition locale ininterrompue, cette notion, qui ne revêt pas un caractère équivoque, est suffisamment précise pour garantir contre le risque d'arbitraire. "

Le Conseil Constitutionnel en tire la conclusion que "le grief tiré de la méconnaissance du principe d'égalité doit être rejeté ; que la première phrase du septième alinéa de l'article 521-1 du code pénal, qui ne méconnaît aucun autre droit ou liberté que la Constitution garantit, doit être déclarée conforme à la Constitution."

Que penser de cette décision?

Il y a deux points qui méritent d'être relevés :

a) Le Conseil dit que l'alinéa 7 ne porte pas atteinte à un droit constitutionnellement garanti. Les sages nous disent que le principe d'égalité issu de l'article 6 de la Déclaration des droits de l'homme et du citoyen de 1789 n'empêche nullement que le législateur puisse régler de façon différente des situations différentes pourvu que la différence de traitement "est en rapport direct avec l'objet de la loi qui l'établit". Dans son commentaire $^{10}$, le Conseil Constitutionnel se réfère à son abondante jurisprudence à ce sujet. Les sages ont vérifié si l'exception est bien en rapport direct avec l'objet de la loi qui l'établit .Pour le Conseil, la réponse est positive car le législateur a introduit une double limite: limite géographique (parties du territoire où existe une tradition locale ininterrompue) et pour les seuls actes qui relèvent de cette tradition. Les sages montrent ici qu'ils n'ont aucunement envie de se substituer au législateur. L'objet de la loi est bien, selon nous, de protéger les animaux contre les actes de cruauté ; or, en instituant une dérogation qui porte atteinte à l'objet de la loi, le Conseil aurait pu en tirer une autre conclusion.

${ }^{10}$ http://www.conseil-constitutionnel.fr/conseil-constitutionnel/root/bank/download/2012271QPCccc_271qpc.pdf 
b) Les sages nous disent que la notion de tradition locale ininterrompue est suffisamement précise pour garantir contre le risque d'arbitraire. Cela est, pour le moins, discutable ainsi que nous le soulignerons plus loin.

Nous relevons au passage que les sages disent autre chose que ce qui est dans la loi. En effet, les sages citent l'alinéa 7 qui exclut de la responsabilité pénale les courses de taureaux lorsqu'une tradition locale ininterrompue peut être invoquée et déclarent que l'exonération pénale est restreinte aux "parties du territoire national où l'existence d'une tradition ininterrompue est établie ". Rappelons que l'alinéa 7 de l'article 521-1 du code pénal distingue entre les courses de taureaux pour lesquelles la tradition locale ininterrompue peut être invoquée et les combats de coqs pour lesquels l'exonération ne s'applique que pour les localités où une tradition ininterrompue peut être établie.

Au-delà de cette remarque, y a deux notions principales qui doivent être examinées par les juges du fond et être réunies pour que l'exonération puisse trouver application :

- La " localité » de la tradition ; la Cour de Cassation dans un arrêt du 10 juin $2004{ }^{11}$ précise que l'adjectif « locale » doit faire l'objet d'une appréciation relativement stricte. La tradition doit exister dans la localité en cause. Les juges du fond dans les régions concernées par l'exonération en font en revanche une interprétation bien plus extensive et donnent souvent à l'adjectif "locale ", une définition bien plus large que celle liée à une localité à proprement parler.

- L'ininterruption de la tradition : la Cour de Cassation dans son arrêt du 10 juin 2004 estimait que cela implique que l'organisation de corridas soit régulière. Cela interdit, dit-elle, de constater cette tradition lorsqu'aucune corrida n'a pas été organisée depuis un grand nombre. Cependant, dans un arrêt du 7 février $2006^{12}$, la première chambre civile de la Cour s'est ensuite livrée à une interprétation bien plus large puisqu'elle a admis qu'une cour d'appel puisse déduire la persistance d'une tradition taurine de " l'intérêt que lui port(e) un nombre suffisant de personnes ". C'est ainsi qu'elle a pu justifier l'existence d'une tradition ininterrompue alors qu'en l'espèce il n'y avait plus eu de corrida à Toulouse depuis 1976 !

11 Cour de cassation, 2ème chambre civile, 10 juin 2004, n 02-17121.

12 Gazette du Palais 26 septembre 2006, n²69, p. 12, note D. Blanc. 
Le fait que l'alinéa 7 de l'article incriminé se référant aux courses de taureaux stipule l'exonération, dès lors qu'une tradition locale ininterrompue peut être invoquée (et donc qui n'a pas à être établie), suffit à fonder une interprétation plus large que si la tradition devait être établie. "Etablir", contrairement à "invoquer" suppose que l'on apporte la preuve de la réalité de l'ininterruption de la tradition locale. Le fait de devoir simplement invoquer une tradition offre dès lors un champ d'application plus large.

Nous ne partageons pas, en conséquence, "l'optimisme" et la confiance du Conseil Constitutionnel lorsque, s'agissant de la tradition locale ininterrompue, il déclare que "cette notion, qui ne revêt pas un caractère équivoque, est suffisamment précise pour garantir contre le risque d'arbitraire".

\section{Les conséquences de la décision.}

La décision rendue par le Conseil Constitutionnel le 21 septembre 2012 a suscité de très nombreuses réactions. Soulagement des pro-corridas d'un côté, volonté clairement affichée par les anti-corridas de l'autre, d'aller jusqu'au bout et d'utiliser toutes les voies juridiques possibles pour aboutir enfin à l'abolition de la corrida en France.

Il est certain que les afficionados se trouvent renforcés dans leurs convictions par la décision rendue.

Pouvons-nous espérer que les choses puissent changer dans un proche avenir ?

Deux propositions de loi ont déjà été enregistrées au bureau de l'Assemblée Nationale antérieurement à la décision du Conseil Constitutionnel, respectivement les 13 juillet $2010^{13}$ et 13 juillet $2011^{14}$ et n'ont toujours pas été débattues à l'Assemblée Nationale.

\footnotetext{
${ }^{13}$ http://www.anticorrida.com/IMG/pdf/PPL_2735.pdf

${ }^{14}$ http://www.anticorrida.com/IMG/pdf/ppl 3695.pdf
} 
Il est d'ailleurs fort peu probable qu'elles soient discutées et adoptées un jour. Il est donc peu vraisemblable que les choses avancent en France par ce biais.

Un grand référendum national souhaité par certains a tout aussi peu de chances d'être organisé un jour même si le résultat serait sans doute favorable à l'abolition comme les montrent les derniers sondages effectués en France. Ainsi, le dernier sondage Ipsos du18-21 septembre $2012^{15}$ a révélé que $48 \%$ des français sont favorables (sauf dans les régions taurines où le chiffre baisse à $39 \%$ ) à l'abolition de la corrida contre $42 \%$ qui y sont favorables .Le journal Midi Libre ${ }^{16}$ avait, de son côté, organisé un sondage sur la ville d'Alès publié sur son site le 24 juillet 2012 qui montre une majorité claire (55\%) en faveur de l'abolition de la corrida, chiffre remarquable lorsque l'on rappelle qu'il s'agit d'une ville taurine parmi les plus éminentes.

On peut aussi rêver à de grandes manifestations publiques auxquelles participeraient des centaines de milliers de personnes y compris dans les régions à tradition tauromachiques qui interpelleraient les femmes et hommes politiques et les amèneraient (peut-être) à reconsidérer leurs positions et à davantage s'impliquer en faveur de l'abolition pour, in fine, voter enfin un texte abolissant la corrida.

On peut également se prendre à rêver à un boycott massif des villes taurines qui leur ferait comprendre qu'il est dans leur intérêt de ne plus autoriser les corridas... Tout cela est cependant très peu probable.

\footnotetext{
${ }^{15}$ http://www.sondages-en-france.fr/post/francais-corrida-ifop-18-20-septembre-2012-1031

${ }^{16}$ http://www.midilibre.fr/2012/07/24/dma-sondage-55-d-alesiens-favorable-a-la-fin-des-corridas,538622.php
} 
L'espoir (le seul ?) consisterait plutôt, ainsi que l'évoque Jean-Pierre Marguénaud dans son article cité antérieurement, à se tourner vers l'Europe. Selon ce dernier, "C'est d'ailleurs essentiellement du côté du droit de la CEDH (Cour Européenne des Droits de I'Homme) qu'elles (les perspectives de remise en cause)pourraient se dégager dans la mesure où le droit de l'Union Européenne a pris un soin méticuleux à subordonner la prise en compte du bien-être des animaux en tant qu'être sensibles au respect des dispositions législatives ou règlementaires et même des usages en matière de traditions culturelles et de patrimoines régionaux auxquels les corridas ne courent aucun risque de na pas appartenir".

Les associations requérantes ont déjà évoqué l'éventualité d'une saisine de la CEDH. Elles le feront sans doute.

Pour notre part, nous estimons qu'une tradition ne mérite d'être maintenue que pour autant qu'elle soit respectable. Or, en l'espèce, la corrida, constituant un acte de cruauté ainsi que le législateur le reconnait implicitement par l'exception qu'il lui reconnait, n'est pas respectable. Une tradition ne doit en aucun cas l'emporter sur la vie qui doit être et rester la valeur suprême, la vie sous toutes ses formes.

Tôt ou tard, nous en sommes convaincus, la corrida disparaitra d'elle-même, faute de spectateurs, faute de rentabilité économique. Malheureusement des milliers de taureaux (en moyenne autour de 1100 par an) tomberont encore dans les arènes d'ici là.

Dossier à suivre de près. 